\title{
Annexin A2 upregulation protects human retinal endothelial cells from oxygen-glucose deprivation injury by activating autophagy
}

\author{
SHULE JIANG ${ }^{1}$ and YILE XU ${ }^{2}$ \\ ${ }^{1}$ Department of Ophthalmology, The First Affiliated Hospital of Zhejiang Chinese Medical University, \\ Hangzhou, Zhejiang 310006; ${ }^{2}$ Department of Ophthalmology, \\ The Hangzhou First People's Hospital, Hangzhou, Zhejiang 310001, P.R. China
}

Received January 30, 2019; Accepted July 30, 2019

DOI: $10.3892 /$ etm.2019.7909

\begin{abstract}
Retinal neovascularization is a common pathological change in multiple diseases of the eyes and the upregulation of annexin A2 (A2) under a hypoxic and ischemic microenvironment has been demonstrated to be a key factor in the pathological process. However, the underlying mechanism by which $\mathrm{A} 2$ regulates retinal neovascularization remains unclear. In the present study, oxygen-glucose deprivation (OGD) was used to mimic the hypoxic and ischemic microenvironment, to observe the role of $\mathrm{A} 2$ in retinal neovascularization regulation by focusing on autophagy. The results showed that OGD treatment significantly increased the mRNA and protein levels of A2 in human retinal endothelial cells (HRECs), which was dependent on activation of hypoxia inducible factor (HIF)- $1 \alpha$ signaling. The OGD-induced activation of autophagy was attenuated when A2 was silenced, but increased when A2 was overexpressed, suggesting that A2 upregulation contributed to OGD-induced cell autophagy activation. Furthermore, knockdown of A2 decreased cell viability and promoted cell apoptosis under OGD conditions. Overexpression of A2 increased cell viability and reduced cell apoptosis under OGD conditions, and inhibiting autophagy using an inhibitor, reversed these changes, suggesting that upregulation of A2 by OGD serves a cytoprotective role by inducing cell autophagy in HRECs. Taken together, the results of the present study suggested that promoting retinal endothelial cell survival by autophagy activation via the HIF- $1 \alpha$ signaling pathway in a hypoxic and ischemic microenvironment may underlie the mechanism by which A2 regulates retinal neovascularization. The present study is the first study to demonstrate the novel role of A2 during retinal neovascularization under pathological conditions, to the best of our knowledge. Therefore,
\end{abstract}

Correspondence to: Professor Yile Xu, Department of Ophthalmology, The Hangzhou First People's Hospital, 216 Huansha Road, Hangzhou, Zhejiang 310001, P.R. China

E-mail: jingzh1234@163.com

Key words: annexin A2, oxygen-glucose deprivation, autophagy, apoptosis, human retinal endothelial cells
A2 may serve as a potential therapeutic target for treating neovascularization-associated conditions of the eye.

\section{Introduction}

Retinal neovascularization is a common pathological change in a number of eye diseases, resulting in severe eye injury and possibly leaving the patient blind $(1,2)$. The upregulation of annexin A2 (A2) under a hypoxic and ischemic microenvironment has been demonstrated to be a key factor in this pathological process (3). It was reported that mice deficient in the $A 2$ gene were resistant to hypoxia-related retinal neovascularization in a model of diabetic retinopathy (3). $A 2^{-/-}$ newborn mice had a reduced neoangiogenic response in the oxygen-induced retinopathy model (4). Additionally, $A 2^{-/-}$mice also displayed reduced angiogenesis in three different models of postnatal angiogenesis (5).

The human A2 gene is located on chromosome 15 (15q21) and is expressed in multiple human cells, including endothelial cells, monocytes, macrophages, dendritic cells, trophoblast cells, and tumor cells $(6,7)$. As a type of $\mathrm{Ca}^{2+}$-regulated, phospholipid-binding protein, A2 exists both as a free monomer in the cytoplasm or tethered to the surface of the plasma membrane (6). Accumulating evidence has demonstrated that A2 plays multifaceted roles in membrane organization, signal transduction, cell proliferation, migration, apoptosis, immunity and inflammation $(3,6,8)$. A2 has also been reported to be closely associated with multiple pathological processes, including thrombosis, vascular occlusion, hyperfibrinolysis, hemorrhage and retinal neovascularization $(3,9)$. Knockdown of the A2 receptor (A2R) significantly inhibited the proliferation, adhesion, migration and tube formation of human umbilical vein endothelial cells (HUVECs), which is usually used in the in vitro study of angiogenesis (10). Disrupting A2R can inhibit neovascularization in vivo via inactivating phosphorylation of the protein kinase B (AKT) and extracellular signal regulated kinase (ERK) signaling pathways (10). The authors' previous study additionally demonstrated that A2 silencing inhibited the proliferation and enhanced the apoptosis of HUVECs (11).

The underlying mechanism by which A2 is required for retinal neovascularization remains largely unknown. In the present study, oxygen-glucose deprivation (OGD) was used to 
mimic the ischemic and hypoxic conditions and the role of A2 in retinal neovascularization and autophagy was observed using human retinal endothelial cells (HRECs). The present study is the first to demonstrate that A2 may promote survival of HRECs under hypoxic and ischemic conditions by inducing autophagy via the hypoxia-inducible transcription factor- $1 \alpha$ (HIF-1 $\alpha$ ) signaling pathway, to the best of our knowledge. The present study also demonstrated a novel role of A2 during retinal neovascularization under pathological conditions and thus highlights a potential therapeutic target for treating conditions where neovascularization of the eye is observed.

\section{Materials and methods}

Chemical reagents and plasmids. A HIF-1 $\alpha$ inhibitor was purchased from Calbiochem (cat. no. 400083; Merck KGaA). The autophagy inhibitor, 3-methyladenine (3-MA), was purchased from Sigma-Aldrich; Merck KGaA. The concentrations of the inhibitors used were determined by previous studies $(12,13)$. The scramble control short hairpin RNA (shRNA) plasmid, A2 shRNA plasmid, the wide-type A2 overexpression plasmid (pcDNA3.1-A2) and control plasmid (pcDNA3.1) were constructed by Shanghai GenePharma Co., Ltd.

Cell culture. Primary HRECs (ACBRI 181) were obtained from Cell Systems and cultured in DMEM (Gibco; Thermo Fisher Scientific, Inc.) supplemented with $20 \%$ fetal bovine serum (Thermo Fisher Scientific, Inc.), $1 \%$ endothelial cell growth supplement (Sigma-Aldrich; Merck KGaA) and $100 \mathrm{U} / \mathrm{ml}$ penicillin and $0.1 \mathrm{mg} / \mathrm{ml}$ streptomycin (Beyotime Institute of Biotechnology) at $37^{\circ} \mathrm{C}$ in a humidified incubator under $5 \% \mathrm{CO}_{2}$. Cells which had been passaged between 10 and 20 times were used for the following experiments.

OGD treatment. At $80-90 \%$ confluence, the culture medium of HRECs was replaced with serum-free and glucose-free DMEM (Gibco; Thermo Fisher Scientific, Inc.), after which the cells were placed in a hypoxic incubator chamber $\left(1 \% \mathrm{O}_{2}\right.$, $5 \% \mathrm{CO}_{2}, 94 \% \mathrm{~N}_{2}$; Forma Scientific; Thermo Fisher Scientific, Inc.) for the indicated times (3, 6, 12 and $24 \mathrm{~h})$. Control cells were cultured with supplemented medium and incubated under normal oxygen concentrations.

$R N A$ extraction and reverse transcription-quantitative $P C R$ (RT-qPCR) analysis. Total RNA was extracted using TRIzol reagent (Invitrogen; Thermo Fisher Scientific, Inc.) and $2 \mathrm{mg}$ total RNA was reverse transcribed using RevertAid ${ }^{\mathrm{TM}}$ First Strand cDNA Synthesis Kit (Fermentas MBI; Thermo Fisher Scientific, Inc.) according to the manufacturer's protocol. The temperature protocol for the reverse transcription was $25^{\circ} \mathrm{C}$ for $10 \mathrm{~min}, 42^{\circ} \mathrm{C}, 60 \mathrm{~min}$ and finally $70^{\circ} \mathrm{C}$ for $10 \mathrm{~min}$. qPCR was performed in triplicate using SYBR Green PCR Master Mix (Toyobo Life Science) on a Mastercycler ep realplex (Eppendorf). The primer sequences used were as follows: A2 forward, 5'-GTGAAGAGGAAAGGAACCGA-3' and reverse 5'-CTTGATGCTCTCCAGCATGT-3'; GAPDH forward, 5'-GCCTTCCGTGTTCCTACC-3' and reverse 5'-AGAGTG GGAGTTGCTGTTG-3'. Thermocycling conditions consisted of an initial denaturing step $\left(95^{\circ} \mathrm{C}, 2 \mathrm{~min}\right)$, followed by 40 cycles of denaturing $\left(95^{\circ} \mathrm{C}, 15 \mathrm{sec}\right)$, annealing $\left(60^{\circ} \mathrm{C}, 15 \mathrm{sec}\right)$ and extending $\left(72^{\circ} \mathrm{C}, 45 \mathrm{sec}\right)$. The mRNA levels were normalized to GAPDH (internal control) and their relative quantities were determined using the $2^{-\Delta \Delta \mathrm{Cq}}$ formula (14).

Western blotting. HRECs were rinsed three times with PBS and lysed in RIPA buffer (cat. no. P0013C; Beyotime Institute of Biotechnology) supplemented with $1 \%$ PMSF to obtain total cell lysates. The protein concentration was qualified using the bicinchoninic acid assay method and $50 \mu \mathrm{g}$ proteins for each sample were separated on a $12 \%$ SDS-PAGE, transferred to PVDF membranes and blocked with 5\% non-fat milk for $60 \mathrm{~min}$ at room temperature. The membranes were probed overnight at $4{ }^{\circ} \mathrm{C}$ with primary antibodies against A2 (cat. no. 8235; 1:1,000; Cell Signaling Technology, Inc.), LC3 (cat. no. 3868; 1:1,000; Cell Signaling Technology, Inc.), Beclin-1 (cat. no. 3495; 1:1,000; Cell Signaling Technology, Inc.), cleaved-caspase 3 (cat. no. 9961; 1:1,000; Cell Signaling Technology, Inc.), HIF-1 $\alpha$ (1:500; Santa Cruz Biotechnology, Inc.) and $\beta$-actin (cat. no. A1978; 1:10,000; Sigma-Aldrich; Merck KGaA). Subsequently, the membranes were incubated with horseradish peroxidase-conjugated secondary antibodies (cat. no. 610-1302; 1:5,000; Rockland Immunochemicals, Inc.) for $2 \mathrm{~h}$ at room temperature. Finally, the blots were visualized using an enhanced chemiluminescence reagent (BeyoECL Plus Kit; cat. no. P0018; Beyotime Institute of Biotechnology). Protein bands were quantified using the ImageJ software (Version 1.52p; National Institute of Health).

Cell transfection. Lentiviral vectors encoding shRNAs against human A2 (sh-A2, sequence 5'-TGAGGGTGACGT TAGCATTAC-3' for A2 shRNA), lentiviral control vectors (vector1, sequence 5'-GGATCATCATGCTATGCAGTT-3' for control scramble shRNA), lentiviral A2 overexpression vectors (OE-A2, primers used as follows, sense: 5'-CGGGGT ACCATGTCTACTGTTCACGAAATCCTGT-3', anti-sense: 5'-ATTGGGCCCGTCATCTCCACCACACAGGTAC-3' and lentiviral control vectors (vector2, pcDNA3.1 for the control plasmid) were packed and obtained from OBiO Technology (Shanghai) Corp., Ltd. HRECs were plated in 24-well plates at a density of $2 \times 10^{5}$ cells/well and allowed to grow at $\sim 40 \%$ confluence. Cells were infected with the aforementioned lentiviral vector (multiplicity of infection, 50) for $48 \mathrm{~h}$ and the transfection efficiency was evaluated by western blotting.

Luciferase assay. The A2 promotor-luciferase reporter and pRL-SV40-luc plasmids were designed and purchased from Promega Corporation. HRECs were plated in triplicate into a 24-well plate at a density of $5 \times 10^{5}$ cells per well for overnight culture, after which the cells in each well were infected with lentivirus particles expressing the reporter plasmid [manufactured by OBiO Technology (Shanghai) Corp., Ltd.]. Additionally, the lentivirus particles expressing pRL-SV40-luc [manufactured by OBiO Technology (Shanghai) Corp., Ltd.] was co-transfected to normalize the transfection efficiency as an internal control (multiplicity of infection, 50). Following transfection for $48 \mathrm{~h}$, cells underwent OGD for another $24 \mathrm{~h}$, in the presence or absence of the HIF-1 $\alpha$ inhibitor $(100 \mu \mathrm{mol} / \mathrm{l})$. Luciferase activity was measured using the dual luciferase assay system (Promega Corporation) and normalized to a constitutively expressed Renilla reporter. 
A

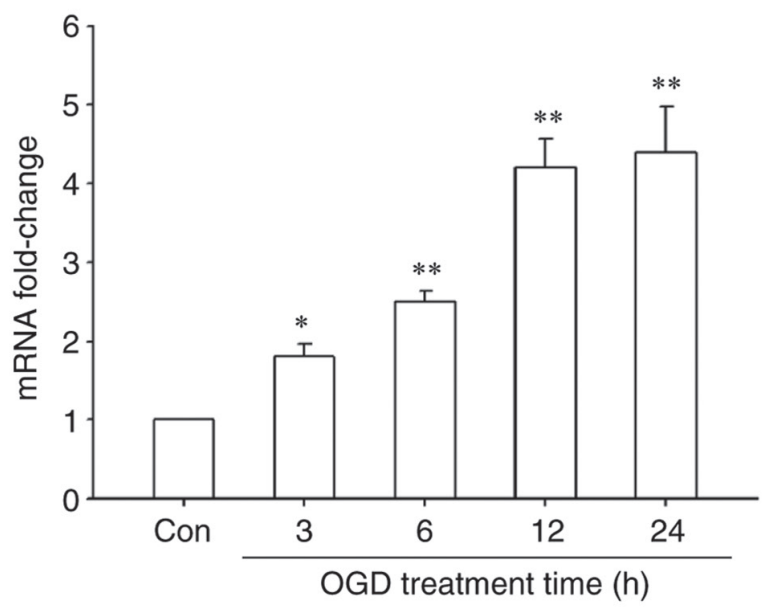

B
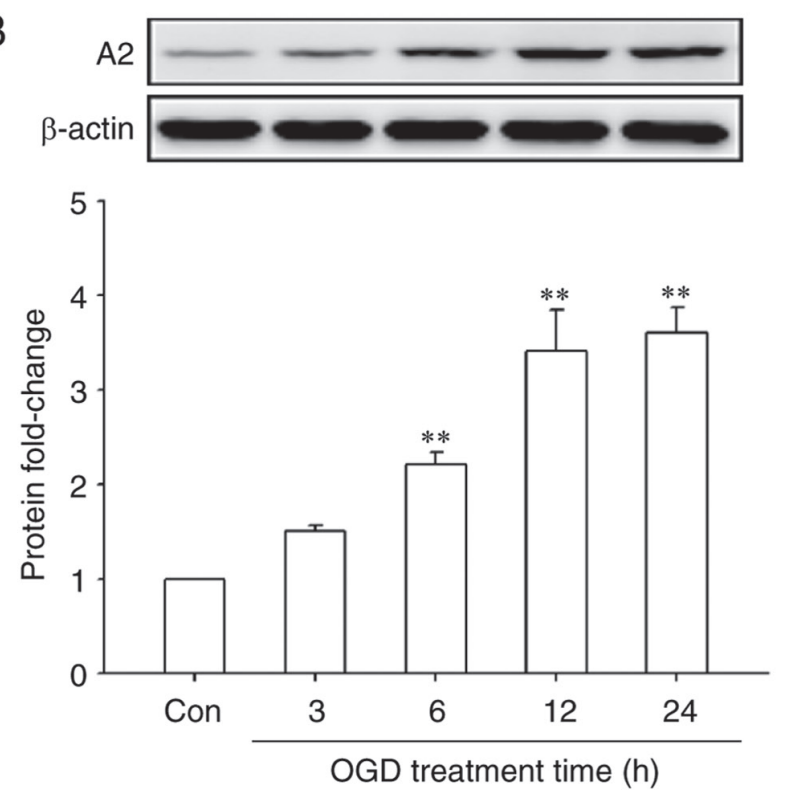

Figure 1. Effects of treatment with OGD for different time periods on the expression of A2 in human retinal endothelial cells. Cells were cultured with serum-free and glucose-free DMEM in a hypoxic incubator chamber $\left(1 \% \mathrm{O}_{2}, 5 \% \mathrm{CO}_{2}\right.$ and $\left.94 \% \mathrm{~N}_{2}\right)$ for the indicated times. Control cells were cultured with complete medium under normal atmospheric conditions. The expression of A2 was detected by (A) reverse transcription-quantitative PCR and (B) western blotting. ${ }^{*} \mathrm{P}<0.05$ and ${ }^{* *} \mathrm{P}<0.01$ vs. Con group. Con, control; OGD, oxygen-glucose deprivation; A2, Annexin A2.

Cell viability assay. HRECs were infected with the lentivirus particles expressing A2-shRNA, A2 overexpressing plasmid or control vector for $48 \mathrm{~h}$ and plated in 96-well plates at the density of $4 \times 10^{4}$ per well in triplicate for overnight culture (multiplicity of infection, 50). Transfected cells underwent OGD for a further $24 \mathrm{~h}$ in the presence or absence of 3-MA (5 mM), after which the cell viability was evaluated using Cell Counting Kit-8 (Dojindo Molecular Technologies, Inc.) according to the manufacturer's protocol. The optical density was measured at a wavelength of $450 \mathrm{~nm}$ using a synergy microplate reader (BioTek China).

Cell apoptosis assay. Cell apoptosis was detected by measuring the protein level of cleaved-caspase 3 as described previously $(15,16)$. HRECs were transfected with A2-shRNA, A2 overexpressing plasmid or control vector for $48 \mathrm{~h}$, after which the transfected cells underwent OGD for another $24 \mathrm{~h}$ in the presence or absence of 3-MA $(5 \mathrm{mM})$. Cell lysates were collected and the protein expression level of cleaved-caspase 3 was determined by western blotting.

Statistical analysis. Statistical significance between multiple experimental groups was analyzed by an analysis of variance with a post-hoc Tukey's test using SPSS statistics version 19.0 software (IBM Corp.). Quantitative data were expressed as the mean \pm standard deviation of at least three experimental repeats. $\mathrm{P}<0.05$ was considered to indicate a statistically significant difference.

\section{Results}

OGD treatment upregulates the expression of A2 in HRECs. First, the effect of OGD treatment on A2 expression in HRECs was investigated by RT-qPCR and western blotting. The results showed that OGD treatment significantly upregulated the mRNA and protein levels of A2 in a time-dependent manner $(\mathrm{P}<0.05$; Fig. 1$)$. The mRNA levels of $\mathrm{A} 2$ began to increase after OGD treatment for $3 \mathrm{~h}$ and reached a maximal significant 4.4-fold increase compared with the control cells after OGD treatment for $24 \mathrm{~h}(\mathrm{P}<0.01$; Fig. 1A). A similar result was observed at the protein level, reaching a maximal significant 3.6-fold increase compared with the control cells after OGD treatment for $24 \mathrm{~h}(\mathrm{P}<0.01$; Fig. 1B). These data indicated that OGD significantly upregulated the expression of A2 in HRECs.

HIF-1 $\alpha$ mediates the upregulation of A2 under OGD conditions. HIF-1 plays a central role in a number of hypoxic events $(17,18)$. Whether HIF-1 also has a role in OGD-induced A2 expression in HRECs was further investigated. As shown in Fig. 2A, OGD treatment for $24 \mathrm{~h}$ significantly increased the expression of HIF-1 $\alpha(\mathrm{P}<0.01)$. Inhibiting HIF-1 $\alpha$ using 400083 significantly inhibited OGD-induced A2 upregulation at both the mRNA and protein levels $(\mathrm{P}<0.01$; Fig. $2 \mathrm{~B}$ and $\mathrm{C}$ ), suggesting that $\mathrm{HIF}-1 \alpha$ regulated the expression of A2 at the transcriptional level. To confirm this speculation, HRECs were transfected with an A2 promoter-luciferase reporter gene and treated by OGD in the presence or absence of a HIF-1 $\alpha$ inhibitor. As shown in Fig. 2D, OGD treatment for $24 \mathrm{~h}$ significantly increased the A2 promoter activity (5.2-fold of control, $\mathrm{P}<0.01$ ), whereas the suppression of HIF-1 $\alpha$ signaling significantly reversed the OGD-induced promoter activity $(\mathrm{P}<0.01)$. These data confirmed that upregulation of A2 by OGD was mediated by transcriptional regulation of HIF- $1 \alpha$ in HRECs.

A2 is required for OGD-induced autophagy in HRECs. Next, the effects of OGD treatment on the activation of autophagy 
A
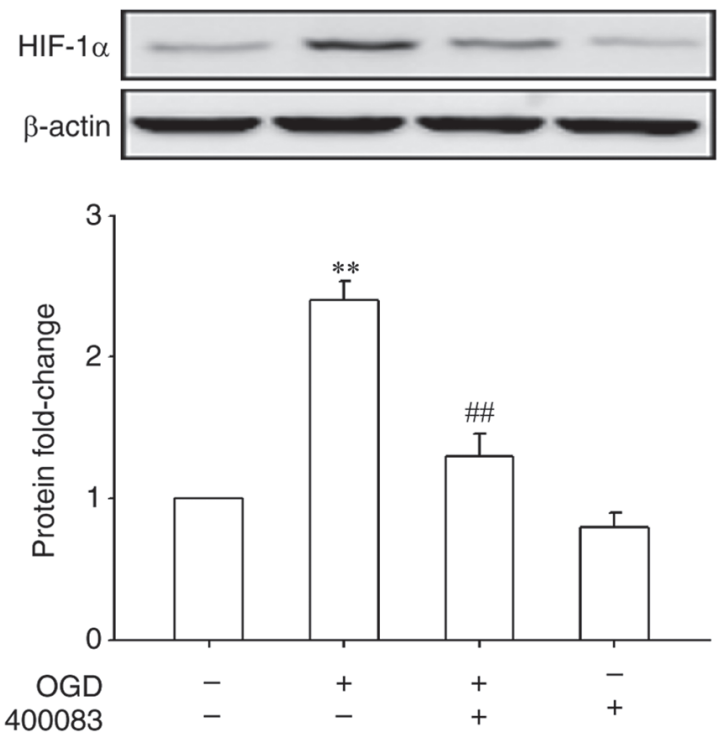

C
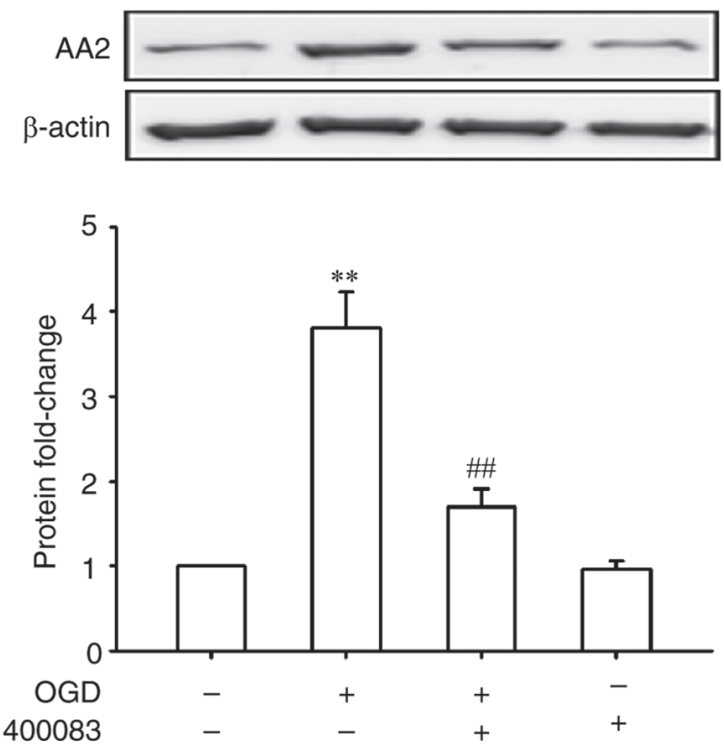
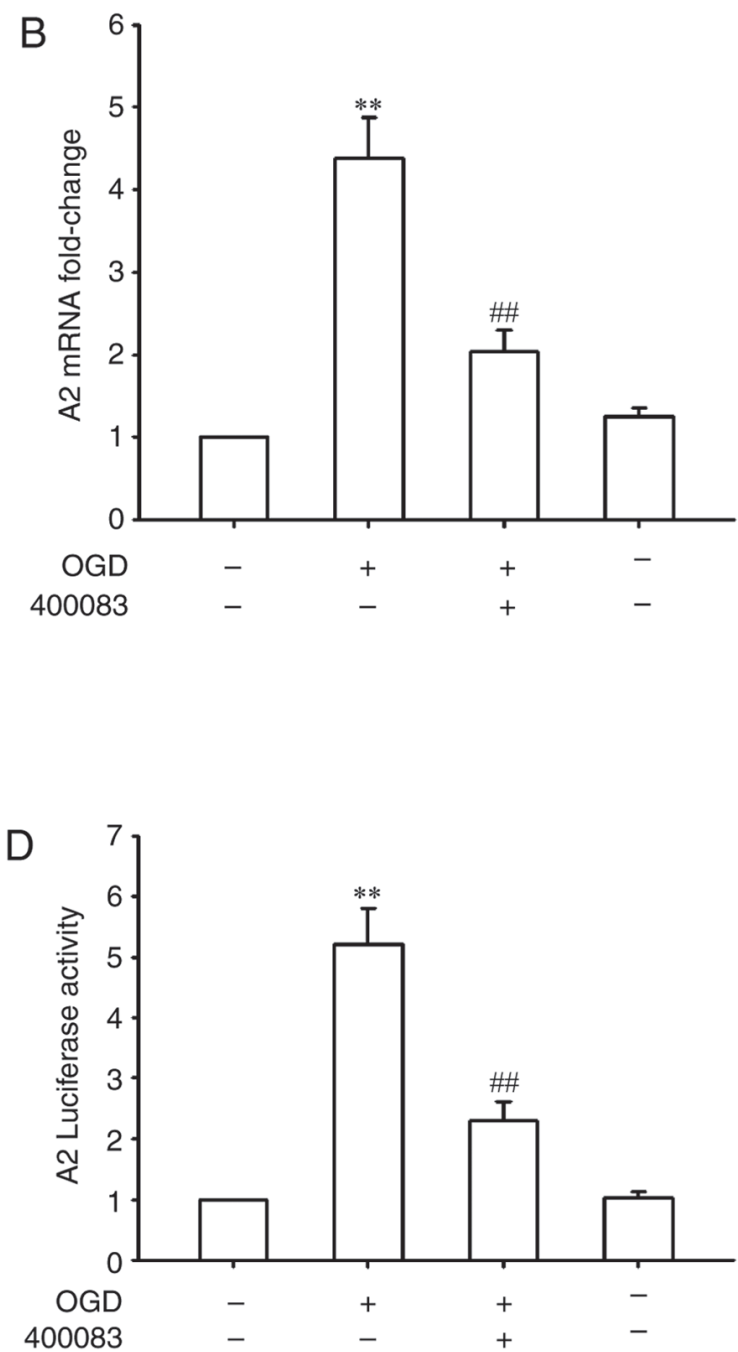

Figure 2. Effects of HIF-1 $\alpha$ inhibition on the expression of A2 in human retinal endothelial cells. The HIF-1 $\alpha$ inhibitor 400083 (100 $\mu$ mol/l) was added to the culture medium immediately prior to OGD treatment. (A) Expression of HIF-1 $\alpha$ detected by western blotting. (B) Expression of A2 detected by reverse transcription-quantitative PCR. (C) Expression of A2 detected by western blotting. (D) A2 luciferase activity. ${ }^{* * *} \mathrm{P}<0.01$ vs. Con group; ${ }^{\# \#} \mathrm{P}<0.01$ vs. OGD group. OGD, oxygen-glucose deprivation; A2, Annexin A2; HIF-1 $\alpha$, hypoxia inducible factor-1 $\alpha$; Con, control.

were observed in HRECs by detecting the expression of Beclin-1 and the conversion of LC3I to LC3II, two usually used markers of autophagy (19), and the role of A2 in this process. The results showed that OGD treatment increased the levels of autophagy in a time-dependent manner in HRECs (Fig. 3A). And, as shown in Fig. 3C and E, OGD-induced autophagy was significantly abrogated after $\mathrm{A} 2$ silencing $(\mathrm{P}<0.01)$ but significantly enhanced by $\mathrm{A} 2$ overexpression $(\mathrm{P}<0.01)$, indicating that A2 upregulation contributed to OGD-induced autophagy activation in OGD-induced autophagy in HRECs. The knockdown and overexpression efficiency of A2 were confirmed by western blotting (Fig. 3B and D).

A2 upregulation protects HRECs against OGD injury through autophagy activation. Finally, whether A2-mediated cell autophagy has a protective effect on OGD injury was observed in HRECs. As shown in Fig. 4, OGD treatment significantly decreased the cell viability and increased the expression of cleaved-caspase 3 (all $\mathrm{P}<0.01$; Fig. $4 \mathrm{~A}$ and B), which was further enhanced after A2 knockdown (all $\mathrm{P}<0.05$; Fig. 4A and B). Overexpression of A2 alleviated these OGD-induced changes, whereas inhibiting cell autophagy with a chemical inhibitor (3-MA) significantly abrogated the pro-survival effects of overexpression of A2 in HRECs $(\mathrm{P}<0.05$; Fig. 4C and D). These results indicated that upregulation of A2 protected HRECs against OGD-induced injury potentially through autophagy activation.

\section{Discussion}

Accumulating evidence highlighted that the role of $\mathrm{A} 2$ in retinal neovascularization, which results in a number of severe 

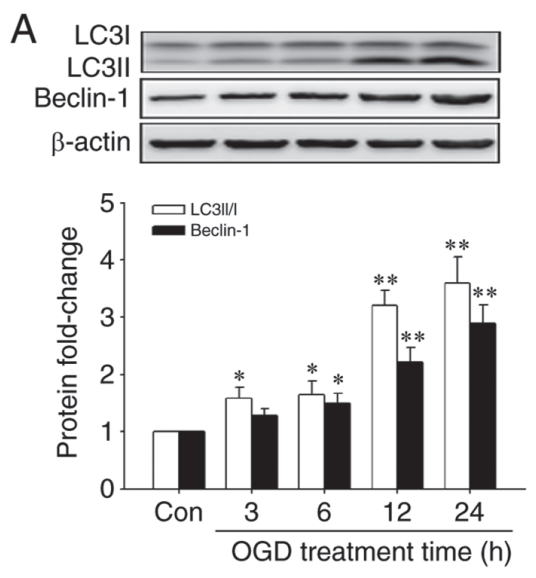

$\mathrm{D}$
B
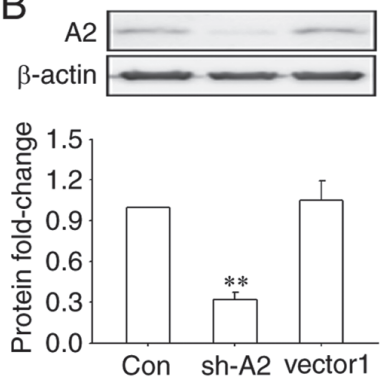
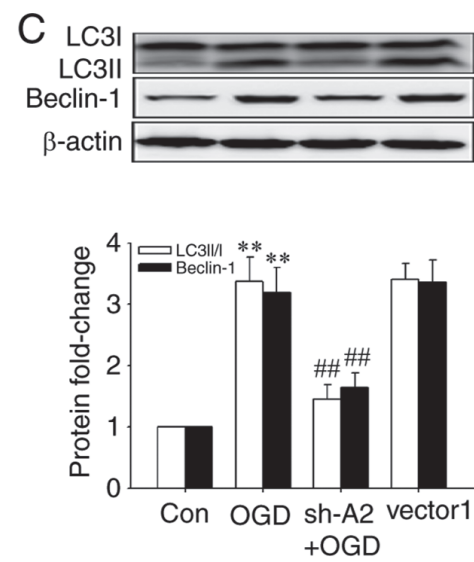

$\mathrm{E}$
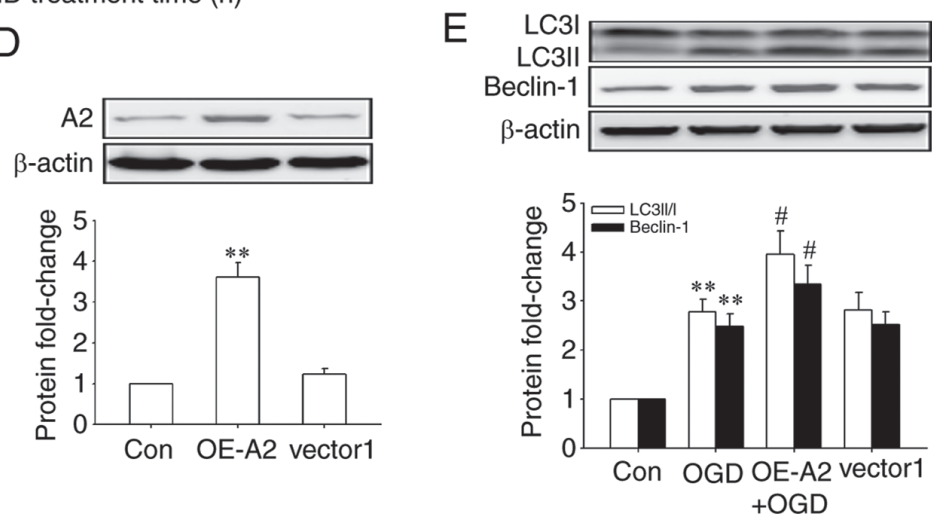

Figure 3. Role of A2 in OGD-induced activation of autophagy in human retinal endothelial cells. Cells were infected with lentiviral vectors encoding shRNAs against human A2, lentiviral scramble control vectors (vector1), lentiviral A2 expression vectors and lentiviral control vectors (vector2) for 48 h before OGD treatment. Protein levels were detected by western blotting. (A) Expression of Beclin-1, LC3I and LC3II when cells underwent OGD for different periods of time. (B) Transfection efficiency of sh-A2. (C) Expression of Beclin-1, LC3I and LC3II following knockdown of A2. (D) Transfection efficiency of OE-A2. (E) Expression of Beclin-1, LC3I and LC3II after A2 overexpression. ${ }^{*} \mathrm{P}<0.05$ and ${ }^{* *} \mathrm{P}<0.01$ vs. Con group; ${ }^{\#} \mathrm{P}<0.05$ and ${ }^{\# \prime} \mathrm{P}<0.01$ vs. OGD group. A2, Annexin A2; sh-A2, lentiviral vectors encoding short hairpin RNAs against human A2; OE-A2, lentiviral A2 overexpression vectors; OGD, oxygen-glucose deprivation; Con, control.

eye diseases and even potentially blindness (3,11,20). Ischemic and hypoxic stimulation are common pathological states in many eye diseases associated with retinal neovascularization. In the present study, OGD was used to mimic the ischemic and hypoxic microenvironment and demonstrated for the first time the expression of A2 was upregulated under OGD conditions both at mRNA and protein levels in HRECs, to the best of our knowledge.

HIF-1 is a heterodimeric protein that consists of HIF-1 $\alpha$ and HIF-1 $\beta$ subunits serves a central role in many hypoxic events $(17,18)$. Hypoxia increases the protein expression level of HIF-1 $\alpha$ by stabilizing it, whereas HIF- $1 \beta$ is constitutively expressed (21). To further determine whether HIF-1 serves a role in OGD-induced A2 expression in HRECs, the effect of OGD treatment on the expression of HIF-1 $\alpha$ and the effect of HIF- $1 \alpha$ inhibition on A2 expression under OGD conditions in HRECs was determined. The results showed that OGD treatment increased the expression of HIF-1 $\alpha$ in HRECs. Inhibiting the HIF-1 $\alpha$ using 400083 decreased the mRNA and protein levels of A2 induced by OGD. To confirm that HIF-1 $\alpha$ regulated A2 expression at the transcriptional level, HRECs were transfected with an A2 promoter-luciferase reporter gene and treated with OGD in the presence or absence of the HIF-1 $\alpha$ inhibitor. The results showed that OGD treatment increased the activity of the A2 promoter, whereas inhibition of HIF-1 $\alpha$ signaling reversed the OGD-induced promoter activity. Together, these data suggest that upregulation of A2 by OGD was mediated by transcriptional regulation of HIF-1 $\alpha$ in HRECs. Previously, Huang et al (22) reported that transcriptional induction of the $A 2$ gene was required in the oxygen-induced retinopathy mice. However, HIF-1 $\alpha$-mediated transcriptional regulation of the $\mathrm{A} 2$ gene requires additional investigation in multiple cell lines.

Regarding the biological significance of A2 upregulation, several studies have reported the role of A2 in regulating autophagy. Li et al (23) demonstrated that A2 positively regulated autophagy in the $\mathrm{MH}-\mathrm{S}$ alveolar macrophages, thereby contributing to host immunity against bacteria through the AKT1-mammalian target of rapamicin-ULK1/2 signaling pathway. Moreau et al (24) showed that A2 modulated the starvation-induced autophagy in HeLa cells and the process was dependent on the A2 effectors, ARP2 and Spire1. Notably, Zhang et al (25) found that overexpression of A2R could also induce autophagy and increase autophagy flux in Mum2C uveal melanoma cells. Consistent with previous studies (23-25), upregulation of A2 contributed to the OGD-induced autophagy in HRECs in the present study, suggesting that A2 can function as an autophagy modulator. However, the underlying mechanism involving A2 in OGD-induced autophagy is still unknown. 
A
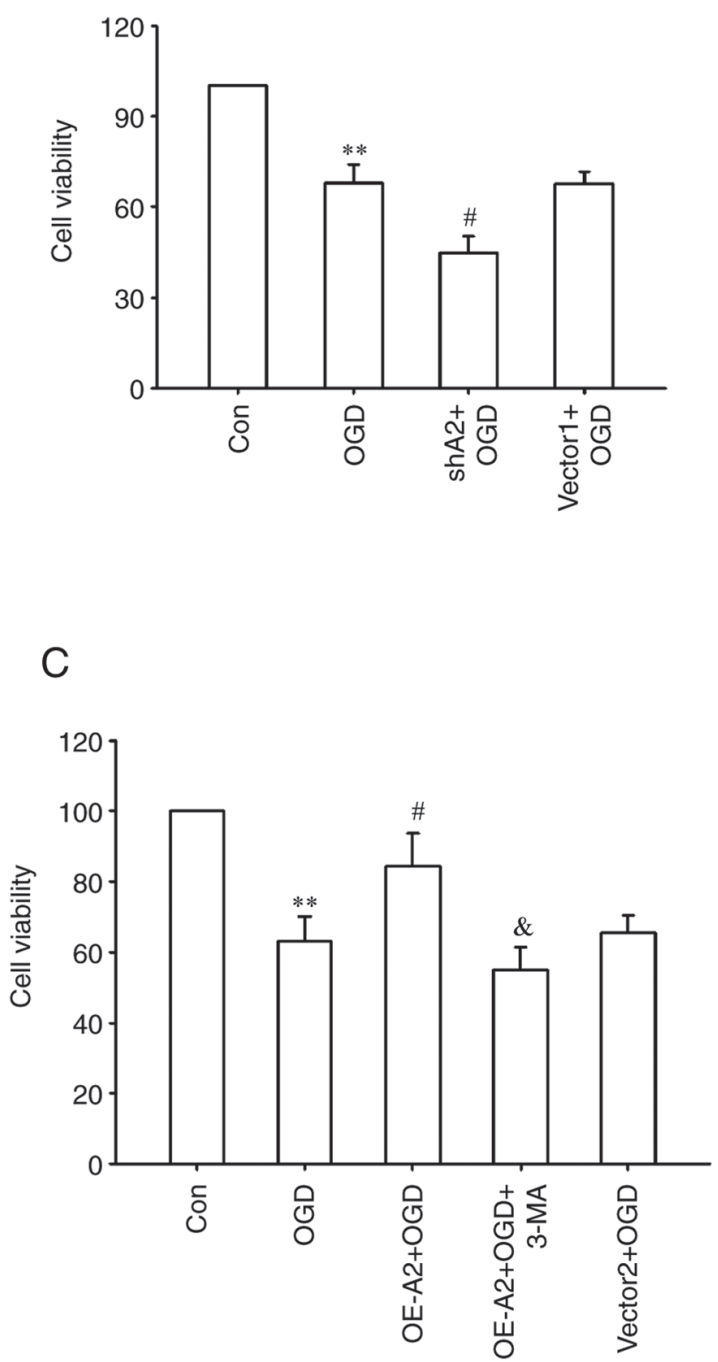
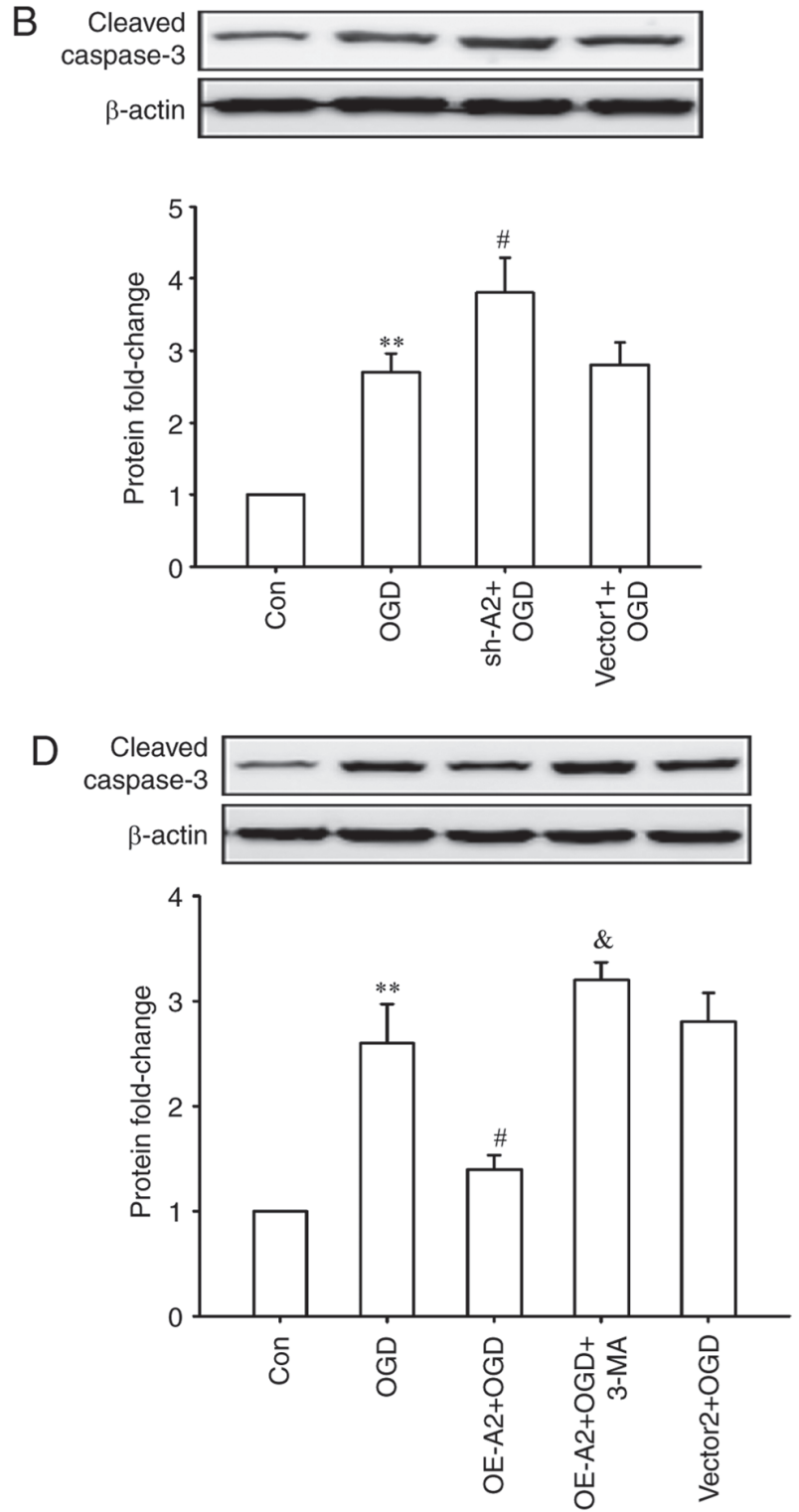

Figure 4. Effects of A2 knockdown or overexpression combined with the autophagy inhibitor 3-MA treatment on OGD-induced injury in human retinal endothelial cells. (A and B) Cells were infected with a lentiviral vector encoding shRNAs against human A2 or a lentiviral scramble control vector (vector 1) for $48 \mathrm{~h}$, then treated with OGD for another $24 \mathrm{~h}$. (A) Cell viability and (B) expression of cleaved-caspase 3 were measured. (C and D) Cells were infected with a lentiviral A2 overexpression vector or a lentiviral control vector (vector 2) for $48 \mathrm{~h}$, then treated with OGD for another $24 \mathrm{~h}$ in the presence or absence of 3-MA $(5 \mathrm{mM})$. (C) Cell viability and (D) expression of cleaved-caspase 3 were measured. ${ }^{* *} \mathrm{P}<0.01$ vs. Con group; ${ }^{*} \mathrm{P}<0.05$ vs. OGD group, ${ }^{\text {\& }} \mathrm{P}<0.05$ vs. OE-A2+OGD group. 3-MA, 3-methyladenine; A2, Annexin A2; OE-A2, lentiviral A2 overexpression vectors; sh-A2, lentiviral vectors encoding short hairpin RNAs against human A2; OGD, oxygen-glucose deprivation; Con, control.

Autophagy is a key mechanism for the maintenance of cellular homeostasis by which damaged organelles and unused proteins are digested and recycled to provide nutrients to promote cell survival (26). A2-regulated autophagy under OGD conditions was associated with cell survival in HRECs. The data in the present study demonstrated that A2 positively regulated autophagy and negatively regulated apoptosis, thereby affecting cell survival. In addition, autophagy inhibition promoted cell apoptosis and decreased cell survival, indicating that A2-regulated autophagy served a cytoprotective role in HRECs under OGD conditions. Previous studies have demonstrated that $\mathrm{A} 2$ could negatively modulate apoptosis in HUVECs and human lung cancer A549, AGZY83-a, Anip973 and BE1 cells $(11,27,28)$. Additionally, studies showed that A2 negatively modulated cell apoptosis through affecting the expression and activity of p53, as well as the downstream proteins in the signaling pathway, such as Bcl2, Bax and the caspase family of proteins $(28,29)$. The results of the present study only demonstrated the anti-apoptotic role of A2 in vitro and the exact role of A2 in regulating endothelial cell apoptosis in vivo remains unknown. The underlying molecular mechanism in which A2 modulated endothelial cell apoptosis also remains unknown. Therefore, further investigations concerning the role of $\mathrm{A} 2$ in apoptosis in gene knock-out mice and its mechanism requires attention.

The roles of vascular endothelial growth factor (VEGF), another HIF-1 $\alpha$ target gene, in angiogenesis had been well studied previously. VEGF promotes cell proliferation, migration 
and tube formation, and regulates cell apoptosis, permeability, metabolism and autophagy in retinal endothelial cells (30-32). A number of studies have reported the role of the interaction of VEGF and A2 signaling and demonstrated that A2 was upregulated by VEGF treatment in monkey retinal vascular endothelial RF/6A cells and osteoblastic MC3T3-E1 cells $(33,34)$. Together, these previous studies and the data presented in the present study suggest that HIF-1 $\alpha / \mathrm{A} 2$ signaling and HIF-1 $\alpha$-regulated autocrine VEGF/A2 signaling may synergistically reinforce neoangiogenesis under ischemic and hypoxic conditions.

In conclusion, the expression of A2 was increased under OGD conditions in HRECs, which is mediated through the activation of the HIF-1 signaling pathway. Upregulation of A2 regulated the activation of cell autophagy and protected HRECs from OGD-induced injury. Therefore, the hypoxic and ischemic environment may trigger the HIF-1/A2 signaling pathway, which can further activate cell autophagy and promote survival of retinal endothelial cells. The data in the present study revealed a novel role and mechanism of A2, which is involved in retinal angiogenesis by regulating autophagy under pathological conditions and provides a potential therapeutic target for treating patients with eye diseases associated with angiogenesis.

\section{Acknowledgements}

Not applicable.

\section{Funding}

No funding was received.

\section{Availability of data and materials}

The datasets used and/or analyzed during the current study are available from the corresponding author on reasonable request.

\section{Authors' contributions}

SJ and YX designed the present study and analyzed the data. SJ performed the experiments.

\section{Ethics approval and consent to participate}

Not applicable.

\section{Patient consent for publication}

Not applicable.

\section{Competing interests}

The authors declare that they have no competing interests.

\section{References}

1. Zhang SX, Ma JH, Bhatta M, Fliesler SJ and Wang JJ: The unfolded protein response in retinal vascular diseases: Implications and therapeutic potential beyond protein folding. Prog Retin Eye Res 45: 111-131, 2015.
2. Mao XB, You ZP, Wu C and Huang J: Potential suppression of the high glucose and insulin-induced retinal neovascularization by Sirtuin 3 in the human retinal endothelial cells. Biochem Biophys Res Commun 482: 341-345, 2017.

3. Hajjar KA: The biology of Annexin A2: From vascular fibrinolysis to innate immunity. Trans Am Clin Climatol Assoc 126: 144-155, 2015.

4. Smith LE, Wesolowski E, McLellan A, Kostyk SK, D'Amato R, Sullivan R and D'Amore PA: Oxygen-induced retinopathy in the mouse. Invest Ophthalmol Vis Sci 35: 101-111, 1994

5. Ling Q, Jacovina AT, Deora A, Febbraio M, Simantov R, Silverstein RL, Hempstead B, Mark WH and Hajjar KA: Annexin II regulates fibrin homeostasis and neoangiogenesis in vivo. J Clin Invest 113: 38-48, 2004.

6. Gerke V, Creutz CE and Moss SE: Annexins: Linking Ca2+ signalling to membrane dynamics. Nat Rev Mol Cell Biol 6: 449-461, 2005.

7. Grindheim AK, Saraste J and Vedeler A: Protein phosphorylation and its role in the regulation of Annexin A2 function. Biochim Biophys Acta Gen Subj 1861: 2515-2529, 2017.

8. Moss SE and Morgan RO: The annexins. Genome Biol 5: 219, 2004.

9. Luo M and Hajjar KA: Annexin A2 system in human biology: Cell surface and beyond. Semin Thromb Hemost 39: 338-346, 2013.

10. Song H, Pan D, Sun W, Gu C, Zhang Y, Zhao P, Qi Z and Zhao S: SiRNA directed against annexin II receptor inhibits angiogenesis via suppressing MMP2 and MMP9 expression. Cell Physiol Biochem 35: 875-884, 2015.

11. Jiang SL, Pan DY, Gu C, Qin HF and Zhao SH: Annexin A2 silencing enhances apoptosis of human umbilical vein endothelial cells in vitro. Asian Pac J Trop Med 8: 952-957, 2015.

12. Huang G, Su J, Zhang M, Jin Y, Wang Y, Zhou P and Lu J: RhoB regulates the function of macrophages in the hypoxia-induced inflammatory response. Cell Mol Immunol 14: 265-275, 2017.

13. Li R, Wang LZ, Du JH, Zhao L and Yao Y: Autophagy activation and the mechanism of retinal microvascular endothelial cells in hypoxia. Int J Ophthalmol 11: 567-574, 2018.

14. Livak KJ and Schmittgen TD: Analysis of relative gene expression data using real-time quantitative PCR and the 2(-Delta Delta C(T)) method. Methods 25: 402-408, 2001.

15. Wu D, Luo N, Wang L, Zhao Z, Bu H, Xu G, Yan Y, Che X, Jiao Z, Zhao T, et al: Hydrogen sulfide ameliorates chronic renal failure in rats by inhibiting apoptosis and inflammation through ROS/MAPK and NF- $\kappa$ B signaling pathways. Sci Rep 7: 455, 2017.

16. You P, Wu H, Deng M, Peng J, Li F and Yang Y: Brevilin A induces apoptosis and autophagy of colon adenocarcinoma cell CT26 via mitochondrial pathway and PI3K/AKT/mTOR inactivation. Biomed Pharmacother 98: 619-625, 2018.

17. Semenza GL: Targeting HIF-1 for cancer therapy. Nat Rev Cancer 3: 721-732, 2003.

18. Riboldi E, Porta C, Morlacchi S, Viola A, Mantovani A and Sica A: Hypoxia-mediated regulation of macrophage functions in pathophysiology. Int Immunol 25: 67-75, 2013.

19. Klionsky DJ, Abdalla FC, Abeliovich H, Abraham RT, Acevedo-Arozena A, Adeli K, Agholme L, Agnello M, Agostinis P, Aguirre-Ghiso JA, et al: Guidelines for the use and interpretation of assays for monitoring autophagy. Autophagy 8: 445-544, 2012.

20. Guo T, Song H, Zhao Z, Qi Z and Zhao S: Overexpression of Annexin A2 receptor inhibits neovascularization via the promotion of krüppel-like transcription factor 2. Cell Physiol Biochem 46: 1617-1627, 2018.

21. Zagórska A and Dulak J: HIF-1: The knowns and unknowns of hypoxia sensing. Acta Biochim Pol 51: 563-585, 2004.

22. Huang B, Deora AB, He KL, Chen K, Sui G, Jacovina AT, Almeida D, Hong P, Burgman P and Hajjar KA: Hypoxia-inducible factor-1 drives annexin A2 system-mediated perivascular fibrin clearance in oxygen-induced retinopathy in mice. Blood 118: 2918-2929, 2011.

23. Li R, Tan S, Yu M, Jundt MC, Zhang S and Wu M: Annexin A2 regulates autophagy in pseudomonas aeruginosa infection through the Akt1-mTOR-ULK1/2 signaling pathway. J Immunol 195: 3901-3911, 2015.

24. Moreau K, Ghislat G, Hochfeld W, Renna M, Zavodszky E, Runwal G, Puri C, Lee S, Siddiqi F, Menzies FM, et al: Transcriptional regulation of Annexin A2 promotes starvation-induced autophagy. Nat Commun 6: 8045, 2015.

25. Zhang Y, Song H, Guo T, Zhu Y, Tang H, Qi Z, Zhao P and Zhao S: Overexpression of Annexin II receptor-induced autophagy protects against apoptosis in uveal melanoma cells. Cancer Biother Radiopharm 31: 145-151, 2016. 
26. Choi Y, Bowman JW and Jung JU: Autophagy during viral infection-a double-edged sword. Nat Rev Microbiol 16: 341-354, 2018.

27. Huang Y, Jin Y, Yan CH, Yu Y, Bai J, Chen F, Zhao YZ and Fu SB: Involvement of Annexin A2 in p53 induced apoptosis in lung cancer. Mol Cell Biochem 309: 117-123, 2008.

28. Wang YX, Lv H, Li ZX, Li C and Wu XY: Effect of shRNA mediated down-regulation of Annexin A2 on biological behavior of human lung adencarcinoma cells A549. Pathol Oncol Res 18: 183-190, 2012.

29. Sharathchandra A, Lal R, Khan D and Das S: Annexin A2 and PSF proteins interact with p53 IRES and regulate translation of p53 mRNA. RNA Biol 9: 1429-1439, 2012.

30. Wang Y, Yang C, Gu Q, Sims M, Gu W, Pfeffer LM and Yue J: KLF4 promotes angiogenesis by activating VEGF signaling in human retinal microvascular endothelial cells. PLoS One 10 e0130341, 2015.

31. Jo DH, Bae J, Chae S, Kim JH, Han JH, Hwang D, Lee SW and Kim JH: Quantitative proteomics reveals $\beta 2$ integrin-mediated cytoskeletal rearrangement in vascular endothelial growth factor (VEGF)-induced retinal vascular hyperpermeability. Mol Cell Proteomics 15: 1681-1691, 2016.
32. Domigan CK, Warren CM, Antanesian V, Happel K, Ziyad S, Lee S, Krall A, Duan L, Torres-Collado AX, Castellani LW, et al: Autocrine VEGF maintains endothelial survival through regulation of metabolism and autophagy. J Cell Sci 128: 2236-2248, 2015.

33. Zhao S, Huang L, Wu J, Zhang Y, Pan D and Liu X: Vascular endothelial growth factor upregulates expression of annexin A2 in vitro and in a mouse model of ischemic retinopathy. Mol Vis 15: 1231-1242, 2009.

34. Genetos DC, Wong A, Watari S and Yellowley CE: Hypoxia increases Annexin A2 expression in osteoblastic cells via VEGF and ERK. Bone 47: 1013-1019, 2010.

This work is licensed under a Creative Commons Attribution-NonCommercial-NoDerivatives 4.0 International (CC BY-NC-ND 4.0) License. 